\title{
Iatrogenic Primary Hypothyroidism
}

National Cancer Institute

\section{Source}

National Cancer Institute. Iatrogenic Primary Hypothyroidism. NCI Thesaurus. Code C131819.

Primary hypothyroidism due to medical or surgical treatment. 